\title{
OPPORTUNITIES TO DEVELOP BASIC MOTOR SKILLS IN VOLLEYBALL AT THE INITIAL STAGE OF EDUCATION
}

\author{
S. Stefanov* \\ Theory and Methodology of Physical Education Department, St. Cyril and St. Methodius University \\ of Veliko Tarnovo, Veliko Turnovo, Bulgaria
}

\begin{abstract}
Most volleyball experts believe that developing basic motor skills and abilities to practice the game of volleyball should begin as early as the age of 8-9. Mini volleyball provides a good opportunity for forming groups of students and organized activities in the first years of school.

THE PURPOSE of this study is to reveal the possibilities for early development of basic motor qualities and skills needed for volleyball.

METHODS: Research, analysis and summary of the specialized scientific methodological literature, pedagogical observation, pedagogical sports experiment.

RESULTS: Dependencies have been established between the development of specific physical qualities for the game of volleyball, specific motor training and the acquisition of basic motor skills in volleyball at the initial stage of elementary school.

CONCLUSION: In order to practice some elements of mini volleyball, good coordination of movements, mastery of technical skills and rational management of movements at a certain speed, pace and rhythm are needed. Mini volleyball provides an opportunity to lower the age limit for starting early volleyball training.
\end{abstract}

Key words: mini volleyball, skills.

\section{INTRODUCTION}

Training high-class athletes in the modern stage of sport development is impossible without taking into account the physical laws and mechanisms of motor activity management. Obtaining and interpreting this knowledge are important both for high sportsmanship and in the training of adolescent athletes.

Along with solving common tasks and improving motor skills, volleyball training at school contributes to the early detection of socalled "volleyball" talents at school age, and is also a prerequisite for large numbers of students of different ages joining regular sports classes not only at school but throughout their lives.

\footnotetext{
*Correspondence to: Svetoslav Stefanov, St. Ciril and st. Methodius University of Veliko Tarnovo, Theory and Methodology of Physical Education Department, 5000 Veliko Turnovo, Bulgaria, Email: stefanov.univt@gmail.com, Mobile: 0878835048
}

Objectives of the study

The huge amount of technical approaches and tactical interactions require players to devote to systematic and long-term training. It is for this reason that we turn our attention primarily to the initial stage of this longstanding creative process and the discovery of fundamentally important motor skills and coordination abilities, which are a prerequisite for mastering volleyball skills as early as the initial stages of elementary school.

Since its inception to the present day, the game of volleyball has been constantly evolving and improving. The need to identify the characteristics and trends for its development concerns many scientists, coaches and activists from countries advanced in volleyball. In Bulgaria, a number of authors such as Gigov (1), Dimitrov (2), Krumova (3), Kiuchukov (4), consider these issues a priority. In a number of their publications and research, as well as in scientific and theoretical analyzes, they explore the problems of volleyball 
training and game improvement. It is seen as a projection of cognitive development and the level of people's physical culture. It manifests social forms of behavior - interaction between participants, preference for partners based on skills, symptoms (5).

Some authors describes the mechanisms of injury in the volleyball practice, which data are valuable for medical insurance planning during a volleyball match, for specialists in sports medicine and for formulation of strategies for prevention of future injuries $(6,7)$.

THE PURPOSE of this study is to reveal the possibilities for early development of basic motor qualities and skills needed for volleyball.

\section{METHODS}

Achieving good results from training and competition is directly related to the effective means, methods and organization of these complex processes. Physical training is aimed at improving swiftness and power qualities, speed, flexibility and jumping endurance. Due to the complex coordination of volleyball movements, learning and improving the technique of the game is a complex and lengthy process. In modern day volleyball, the skill to play at high speeds is becoming increasingly important. The ability to control the body and to control the movements in unsupported position needs to be developed at an early stage of learning. That is why our efforts were focused on creating a promising methodology for mini volleyball, which includes developing a set of good motor skills that come along with the diversity of moves in the game of volleyball. One of the specific abilities that young volleyball players need to develop is to determine the trajectory and speed of flight of the ball aimed at them, and to move and take the necessary starting position to play. The so-called anticipation allows them to position themselves ahead of time and to act rationally.

Due to the complex nature of volleyball as a game, in terms of its technical and tactical specifics and the high requirements for its practitioners regarding physical abilities, longterm sports training is required for successful performance in competitions $(8,9)$. Most volleyball experts believe that the development of basic motor skills should begin at the age of 8-9 and it is the practice of the game of mini volleyball at this age that provides a good opportunity to organize students in groups and organized activities. Volleyball takes an important place among the great variety of means of physical education. It is a core discipline of school education curricula and one of the most popular games in school (10).

The rich content of the game is the basis for the development of both basic physical qualities and game-specific qualities and habits. There is imperceptible improvement in coordination of movements, the functions of the neuromuscular apparatus, also joints are strengthened and changes in the functions and work of the analyzers occur.

\section{METHODS}

Nature of the game and ideas embedded in mini volleyball as a sport game at the initial stage of education.

Starting with mini volleyball training at an early age leads to a timely introduction to the basic techniques, laying the foundations for fundamental motor qualities, creating a good basis for motor skills and later on their improvement at a higher level.

In our opinion, the introduction of mini volleyball in the system of physical education in school will help the process of increasing the effectiveness of training, in particular giving extra time as compared to the current distribution of early discovery of prospective practitioners. Practicing mini volleyball is unthinkable without first mastering the technique of performing the basic elements. The basis for successful actions are rapid movement, high rebounds, various strokes on the ball. At the same time, technical elements are performed within very brief contact with the ball.

\section{Opportunities to create a motor basis of specific skills for volleyball}

Among the main basic components of volleyball are spatial orientations, ability to balance, ability to differentiate the parameters of movement. Playing helps improve all body functions. Constant interaction with the ball assists the development of deep and peripheral vision; the reactions to visual and auditory stimuli are improved. During the game the participants show positive emotions cheerfulness, liveliness, initiative.

\section{The basic motor skills for mini volleyball practitioners are:}


1. To move along the court, assessing the direction of the ball and whether it will fall on the court or go out.

2. To develop a good sense of balance and to have stable behavior on the field, which will allow deft, fast and safe movements.

3. To predict the direction of the ball thrown by the opponent, while staying within the playing field (for example, the net and the lines).

4. To deal with the rules of mini volleyball and the simplified point system.

The scientifically based methodology for mini volleyball training, developed and tested by us, provides opportunities for development of basic motor qualities and habits, typical for volleyball at this age, which in our opinion provides basis for formation of specific skills in the next level of training. All this corresponds to the goals we set in our work during the sport experiment and is laid down as basis for our observations during mini volleyball classes.

\section{Goals we set by practicing the game of mini volleyball:}

1. Maximum increase in the number of touches to the ball by one participant while exercising with the purpose of improving technical skills. Exercises 1-1, 2-2.

2. Maximum diversification of the trajectory of the ball, which reaches each participant before applying a given technical approach. Each touch of the ball must be preceded by movement on the court.

3. Activities during the game must be realistic to meet the level of technical preparedness so that children are not disappointed with the handling of the ball. Children must first learn to catch the ball and throw, and then to perform a volleyball pass.

4. During the game, participants are given a greater chance to control the ball, and they can be given a chance to catch it in one of the three touches permitted by the rules before it is thrown over the net. It is to be avoided that the players remain in a passive position.

5. Various technical elements of volleyball approaches are included. They are adapted to the age group of the players. The adapted technique causes children to feel satisfied with their performance during the game.

The expected results from the initial mini volleyball training can be summarized as:

1. Developing the skill to pass and catch the ball on the spot and after moving, after a self- throw, after a pass from a partner and after receiving a ball coming from the opponent's field, as well as the ability to transfer the ball to the opponent's field.

2. Proficiency in the "Mini 4" level of the game, knowledge and successful interpretation of basic rules of the game and application of the classification developed by the FIVB in the "Level 3"game.

3. Skills to move in the field and perform basic elements of feeding with two hands from below and lower frontal initial blow.

4. Mastering the Mini 3 and Mini 4 levels of the game by practising the game at Level 4 of the classification.

5. The skill to pass with two hands from above after a self-throw or after a pass from a partner. Subject, object and target group of the research

The subject of the research is the mini volleyball training methodology at the initial stage of elementary education.

The object of this study is the influence of the applied methodology on the specific physical qualities and basic motor skills.

The target group of research is 90 students from "Emilian Stanev" and "Vladimir Komarov" schools in Veliko Tarnovo, aged 910 years and involved in mini volleyball classes under the project "SUCCESS" of the Ministry of Education, Youth and Science.

\section{RESULTS}

The methodology applied to the experimental group includes a system of special exercises aimed at improving accuracy by using different circumstances for their implementation. The development of each skill as part of the children's preparation is carried out through instructions for performing a specific action or amplitude or repeating a precisely defined form of the exercise.

As early as the preparatory part of the lesson there is an emphasis on the absolute accuracy of performing the exercises (the level of gaze, the direction of the arms, legs, etc.). At the same time, the lesson is of complex character, including a variety of exercises to develop motor and coordination skills, games, implementation of humor, jokes, vivid comparisons, all this allowing a positive atmosphere, raising the emotional state of participants, which helps the normal course of neurological processes and faster mastering of the appointed exercises. 
The main part of the lesson uses exercises to develop motor memory (more complex coordination exercises for attention and reproduction of motor activities). These exercises are repeated throughout the class period, along with performing structurally different motor tasks, which allow to alternate high concentration of attention with relaxation and rest, i.e the pace of the game of volleyball is mimicked, where such phases are constantly alternating.

The final part includes breathing recovery games, which are also assessed for accuracy.

The accuracy of each movement is determined by the degree of compliance with the visual demonstration. Motor actions which require concentration of effort for a short time, are quickly and easily mastered with high enough level of accuracy, and such are most of the technical approaches of volleyball, which are performed in simplified conditions.

The experimental group is given tasks for directing and rhythmically managing the movements, and for mastering the rhythm of a given exercise the main moment of application of the effort is determined, which is separated from the preparatory and final actions. For example, for an initial stroke, the emphasis is on hitting the ball, which is segmented by approach, rebound and landing.

This makes it possible to control several motor actions at the same time, which are externally perceived as a whole, as a natural flow of movements.

When performing the motor actions of the experimental group, attention is paid to compliance with the following conditions:

- degree of compliance with the implementation model;

- the number of repetitions to master the technique of the studied exercise;

- the quality of new motor tasks of varying complexity, performed over a period of time.

The mini volleyball program is designed as a two-year training cycle, with the first cycle featuring a game with mini volleyball rules at the Mini 3 level and the second at the Mini 4 level.

In the initial stages of training with adolescents, different versions of the game technique are used to ensure that beginners will have fun while playing. The goal is to avoid tension, fear, stiffness when performing exercises of special preparatory nature for mastering the rules and techniques of mini volleyball.

During the game it is important to avoid at all costs:

- Time spent without a ball in hand.

- Monotonous warm-up without a ball (children want to play immediately).

The new concepts needed for the normal course of classes are essential, but they must be explained outside of training time.

The following points are essential in the learning process:

- Each participant in the exercises is to receive a ball in order to immediately build his confidence.

- The sports educator is to create basic game situations and then gradually include volleyball-specific exercises.

- Exercises should be short and understandable to eliminate the risk of boredom.

The physical education teacher can compose an unlimited number of sets of exercises to develop a feel for the moving ball, taking into account the degree of complexity and accessibility of the exercises and periodically changing the movements involved.

The concept of basic motor skills is in correlation with other related terms such as coordination of movements, motor agility, and rhythm of movement.

The variety of movements in volleyball suggests that an array of motor situations need to be taken into consideration.

The methodology is adapted for initial mini volleyball training in the elementary school years, based on the requirements of the International Volleyball Federation, in compliance with the recommendations for the development of mini volleyball in the world. It mainly includes exercises developed to focus on moving in space. Such movements are different types of running, walking, jumping, which precede different volleyball techniques. The basis of the methodology is the consideration of the moment of determining the place and moment of contact with the ball and the moment of separation of the ball from the hands.

The purpose of the means used is to develop motor habits and skills that are fundamental for the game of volleyball as early as the initial 
stage of elementary education and to create a basis for their maintenance and stabilization for a long enough period of time. Formation of rational motor structures and accumulation of motor potential in adolescents is one of the main goals of our methodology. Its constituent parts are training and improvement of movements. Learning manifests itself as development of skills. Here it is very important to determine when the training is over and when refinement starts. Each repetition should not be considered as a repetition, but as a new level of awareness of motor habit and improvement through analysis of peripherally incoming information.

In mini volleyball, there should not be too many restrictions imposed on the trainees. The freedom of action and the use of the individual characteristics of the participants provide a solid basis for proceeding towards sports achievements afterward. Unlike other sports, mini volleyball cannot be practiced before mastering the technique of the basic elements. Without them, the ball is difficult to hold in the air, which is the main feature of the game. The basis of successful actions in mini volleyball is fast movements, frequent and high bounces and various strokes on the ball. Brief contact with the ball when performing technical approaches is typical for the game, noting the lack of time to process the ball. This is why it is necessary to immediately and correctly assess the situation, to have a rapid visual and motor reaction and the ability to control several objects simultaneously.

\section{CONCLUSION}

The formation of motor habits for the game begins with the accumulation of specific physical qualities through physical training, which includes a set of exercises for the development of specific strength, speed, agility, endurance, bounce.

In the process of training, mini volleyball players must learn to:

- Coordinate their movements with the trajectory and speed of the ball.

- Take into account the location of the players on the field (both teammates and the opponent).

- Anticipate the actions of other players.
- Quickly analyze the current situation in the game.

- Decide on the most appropriate action.

- Effectively perform technical approaches.

The main accents in the technique of the game mini volleyball are:

- Moving with a ball and timely execution of various technical elements.

- Finding the best starting position to make contact with the ball.

- Using the strength of the arms and the whole body to feed with a higher or lower trajectory.

- Exercises with a partner in pairs.

- Games incorporating catching the ball and throwing between two students;

- Mastering ball grip plus pass or pass plus catch.

\section{REFERENCES}

1. Gigov, D., Education and training in volleyball tactics, Myth, Sofia, pp. 67-69, 1988.

2. Dimitrov, M., Current problems of volleyball, Myth, Sofia, pp. 33, 1996.

3. Krumova, A., Defense in volleyball, MiF, S., pp. 78, 1979.

4. Kyuchukov, B., Improving the complex reactions when playing in attack in volleyball, VFK, 1987.

5. Palladino, L.,(2000) Technical and didactic organization of the young miniature volleyball sector in the Allievi category.

6. Belomazheva-Dimitrova, S., Angelova, P., Frequency and epidemiology of traumas in female student volleyball players. Pedagogical Almanac, Issue 2, pp. 279285, 2019.

7. Dyakova, G., Angelova, P., Angelova, Iv., Dyakov, T., Belomazheva-Dimitrova, S., Sports injuries in students - athletes. Trakia Journal of Sciences, Vol. 15, Suppl. 1, pp 369-374, 2017.

8. Bozhkova, A., Improving the training process in volleyball in non-specialized sports schools, Dissertation, NSA, Sofia, 2007.

9. Kostov, K., Methodology of teaching physical education in grades 1-3 of ESPU, Blagoevgrad, 1987.

10.10.www.//192.190.66.44/documents/A/F/4/

\{AF436255-92BC-4532-9964

D56A0EA380EE\}ti249.pdf06]. 